\title{
The top quark width constrains from the single top quark pro- duction channel.
}

\author{
Alexey Baskakov ${ }^{1, \star}$, Eduard Boos ${ }^{12, \star \star}$, and Lev Dudko ${ }^{1, \star \star \star}$ \\ ${ }^{1}$ Skobeltsyn Institute of Nuclear Physics M.V. Lomonosov Moscow State University, Moscow 119991, Rus- \\ sian Federation \\ ${ }^{2}$ Faculty of Physics M.V.Lomonosov Moscow State University Leninskie Gory, Moscow 119991, Russian \\ Federation
}

\begin{abstract}
Using model independent and gauge invariant method proposed in previous studies we provide estimations on the top quark width measurement from the single top quark production channel and discuss the results of combining measurements from single and pair top quark production.
\end{abstract}

\section{Introduction}

Recently, the topic of improving the accuracy of the direct measurement of the top quark width has received a new impetus for development [1-5]. This interest was mainly motivated by idea of the Higgs boson width measurement in non-resonant region [6-8]. Due to differences between Higgs boson and top quark production mechanisms this idea could not be directly applied to the top quark sector and requires additional study. This study extends the application of the previously proposed method [4] to the case of the single top quark production. Numerical estimations on the top quark width value from the single quark top t-channel production are obtained.

\section{Numerical estimations}

In the previous study [4] we considered complete tree-level process $p p \rightarrow W^{+} W^{-} b \bar{b}$. The phase space of this process was divided for three different kinematical regions: double-resonant (DR), singleresonant (SR) and non-resonant (NR), relative to the resonant peak of the top quark production. The top quark width value was parametrized with two parameters $\Gamma_{\mathrm{t}}=\xi^{2} \cdot \Gamma_{\mathrm{t}}^{S M}+\delta \cdot \Gamma_{\mathrm{t}}^{S M}, \xi$ and $\delta$ cannot be combined into one parameter, as $\xi$ affects $W t b$ coupling as well. These parameters reflect the different nature of possible deviations from the SM value of the top quark width $\Gamma_{t}^{S M}$. For the convenience of further evaluations, it is convenient to perform a replacement $\epsilon=\xi^{2}-1$, so that both parameters $\epsilon$ and $\delta$ are equal to zero in the SM case. It was shown that different kinematical regions have a fundamentally different dependence on the $\epsilon$ and $\delta$ parameters. DR region is mostly sensitive to the $\delta$ parameter as it directly affects top quark width in the resonant peak, while NR region is strongly addicted with $\epsilon$ changes, because of its impact to the $W t b$ vertex.

\footnotetext{
$\star$ e-mail: a.baskakov@cern.ch

$\star \star$ e-mail: boos@theory.sinp.msu.ru

$\star \star \star$ e-mail: lev.doudko@cern.ch
} 


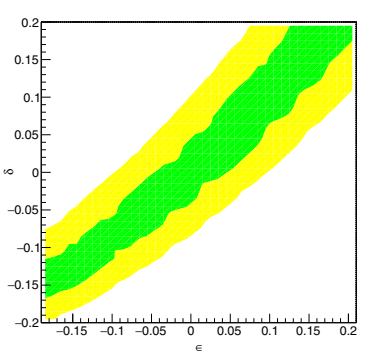

(a) SR region

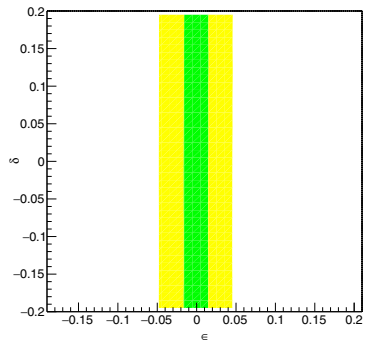

(b) NR region

Figure 1. Constraints on the $\epsilon$ and $\delta$ parameters from the t-channel CS for the $14 \mathrm{TeV}$ collision energy measured with 5\% uncertainty. Green and yellow arias correspond to exclusion limits at $68 \%$ and $95 \%$ CL.

In this study we provide estimations on the top quark width measurement from t-channel single top quark production process $\mathrm{pp} \rightarrow \mathrm{W}^{+} \mathrm{bq}$, where q corresponds for the lite quark u, $\mathrm{u}, \mathrm{d}, \overline{\mathrm{d}}, \mathrm{c}, \overline{\mathrm{c}}, \mathrm{s}, \overline{\mathrm{s}}$. It is known as "five-flavor scheme" (5FS) when a b-quark is involved in the initial state. For consistency with the previous analysis, similar input setup was used, namely, the CompHEP generator [9] with MSTW2008 PDF [10], top quark mass was taken to be $m_{\mathrm{t}}=172.5 \mathrm{GeV}$ and the LO SM value of the top quark width was taken to be $\Gamma_{\mathrm{t}}^{S M}=1.49 \mathrm{GeV}$. Resonant region is defined within $15 \Gamma_{\mathrm{t}}^{S M}$ in the vicinity of $m_{\mathrm{t}}$ value of the invariant $W b$ mass distribution.

In comparison to the $t \bar{t}+t W$ process $\mathrm{pp} \rightarrow \mathrm{W}^{+} \mathrm{W}^{-} \mathrm{b} \overline{\mathrm{b}}$ where $\mathrm{DR}, \mathrm{SR}$ and NR regions can be isolated, the phase space of the single top quark production process contains only SR and NR regions. Contours for the SR and NR regions are shown in the Fig. 1 (a) and (b) respectively. It should be noted that the dependence on $\epsilon$ and $\delta$ of the relevant kinematic regions for the $\mathrm{pp} \rightarrow \mathrm{W}^{+} \mathrm{W}^{-} \mathrm{b} \overline{\mathrm{b}}$ and $\mathrm{pp} \rightarrow$ $\mathrm{W}^{+}$bq processes is very similar. It was also checked that in case of the "four-flavor scheme" (4FS) all main dependencies on $\epsilon$ and $\delta$ parameters are not changed. Contour Fig. 2 (a) shows combined limit from this two regions. Lack of the DR region complicates obtaining restrictions on the $\delta$ parameter, as it mostly affects resonant region. In this case single top quark production channel may allow to improve constraints on the top quark width only if its experimental uncertainty of the fiducial cross section measurement will be significantly lower than in $t \bar{t}+t W$ process. During study of the $t \bar{t}+t W$ process feasible accuracy of $10 \%, 8 \%$ and $5 \%$ for 14,28 and $100 \mathrm{TeV}$ collision energies were assumed. To illustrate possible improvements from the single top channel experimental uncertainty where taken as $5 \%, 3 \%$ and $2 \%$ for 14,28 and $100 \mathrm{TeV}$ Fig. 2. This rough assumption allows to estimate the achievable constraints on the top quark width from the single top quark production from $14 \%$ to $5 \%$ for the energies from 14 to $100 \mathrm{TeV}$ with assumed experimental accuracy of fiducial cross section measurements from $5 \%$ to $2 \%$.

\section{Results}

The ability to obtain direct restrictions on the top quark width from the single top t-channel production on the LHC has been studied. It was shown that single-resonant and non-resonant regions in case of single top quark productions have similar dependences on $\epsilon$ and $\delta$ parameters, as in case of the $t \bar{t}+t W$ process. Improvement of the top quark width limitations can be obtained only if the systematic uncertainty of single top quark cross section would be significantly lower than for the $t \bar{t}+t W$ process. 


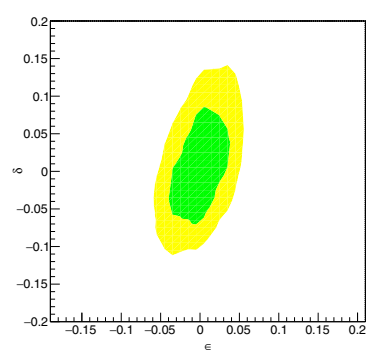

(a) $14 \mathrm{TeV}$

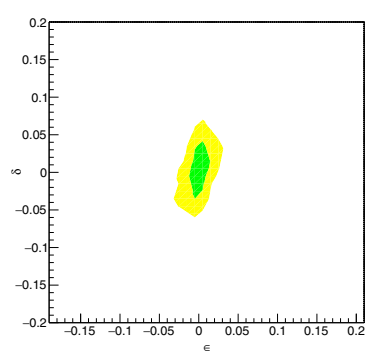

(b) $28 \mathrm{TeV}$

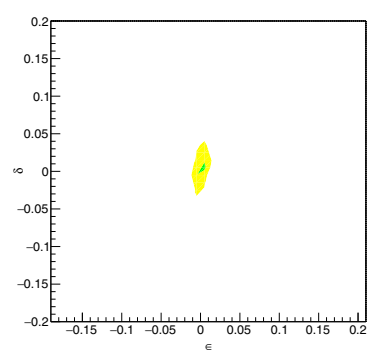

(c) $100 \mathrm{TeV}$

Figure 2. Combined constraints on the $\epsilon$ and $\delta$ parameters for SR and NR regions from the t-channel single top quark production for different collision energies. Green and yellow arias correspond to exclusion limits at $68 \%$ and $95 \% \mathrm{CL}$.

\section{Acknowledgments}

The work was supported by grant 16-12-10280 of Russian Science Foundation.

\section{References}

[1] S. Liebler, G. Moortgat-Pick, A.S. Papanastasiou, JHEP 03, 099 (2016), 1511.02350

[2] P.P. Giardino, C. Zhang, Phys. Rev. D96, 011901 (2017), 1702.06996

[3] A. Baskakov, E. Boos, L. Dudko, EPJ Web Conf. 158, 04007 (2017)

[4] A. Baskakov, E. Boos, L. Dudko, Phys. Rev. D98, 116011 (2018), 1807.11193

[5] C. Herwig, T. Ježo, B. Nachman, Phys. Rev. Lett. 122, 231803 (2019), 1903.10519

[6] N. Kauer, G. Passarino, JHEP 08, 116 (2012), 1206.4803

[7] F. Caola, K. Melnikov, Phys. Rev. D88, 054024 (2013), 1307.4935

[8] J.M. Campbell, R.K. Ellis, C. Williams, JHEP 04, 060 (2014), 1311. 3589

[9] E. Boos, V. Bunichev, M. Dubinin, L. Dudko, V. Ilyin, A. Kryukov, V. Edneral, V. Savrin, A. Semenov, A. Sherstnev (CompHEP), Nucl. Instrum. Meth. A534, 250 (2004), hep-ph/0403113

[10] A.D. Martin, W.J. Stirling, R.S. Thorne, G. Watt, Eur. Phys. J. C63, 189 (2009), 0901.0002 